\title{
Vortex Problems, Rotating Spiral Structures, and the Hannay-Berry Phase
}

\author{
Paul K. Newton and Banavara Shashikanth \\ Department of Aerospace Engineering \\ University of Southern California \\ Los Angeles, CA 90089-1191, USA
}

\begin{abstract}
This paper describes the occurence of phase anholonomies in the context of point vortex problems for two-dimensional incompressible flows. After giving a brief description of anholonomic effects in other contexts, we focus attention on the restricted three-vortex problem and a simpler modified problem where the 'Hannay-Berry' phase can be computed using multi-scale asymptotic methods. Our main emphasis in this paper is to show how the Hannay-Berry phase arises as the leading term in an asymptotic expansion as the result of a non-uniform limit process. We show how it arises when computing the long time growth rate of passive scalar interfaces as they wrap around vortex cores in the presence of a slowly varying background field due to other vortices, and discuss the results in the context of 'spiral-vortex' models for $2 \mathrm{D}$ turbulence.
\end{abstract}

\section{Introduction}

Anholonomic effects arise in mechanical systems both on the classical as well as quantum levels. Simply put, anholonomy is the failure of a system to return to its final configuration during a cyclic change in the systems variables. As a simple example, stand upright with your right arm hanging down, palm forward. Keeping your arm straight, move it up through 90 degrees in the plane of your body so that it is now parallel to the floor. Next, rotate it forward through 90 degrees so that it points in front of you. Finally, let your arm drop back to your side. Your palm should now be facing your body, a 90 degree rotation from its initial configuration, despite the fact that your arm has completed one full cycle [12]. A similar anholonomy occurs when a cat is dropped from an upsidedown position and has to re-orient itself before coming in contact with the floor [12], and when a Foucault pendulum oscillates through a 24 hour period as long as it is not situated on the Equator or one of the Poles [5, 6].

Phase anholonomies have become the focus of intensive research since the discovery of such an effect in quantum mechanics by M. Berry in 1984 [2, 3, 4]. Classical analogues were then discovered by J. Hannay [10], and since that time, several review papers have been written and many new insights have been gained $[8,12,18]$. Our purpose in this paper is to describe how such phase anholonomies arise in vortex dynamics problems, and in particular how they play an important role in determining the long time growth rates of passive scalar interfaces in two-dimensional turbulence problems. 


\section{The Berry phase as a non-uniform limit process}

The Hannay-Berry phase for the restricted 3 vortex problem was treated in detail in [15]. The basic configuration, shown in Fig. 1, has two co-rotating point vortices of equal strength a distance $D$ apart, with a tracer particle orbiting around one of the point vortices with coordinates $(r, \theta)$ from the vortex center. In this problem, we are interested in the particle motion in the limit $D \rightarrow \infty$. In these coordinates, the (normalized) particle equation is given by:

$$
\begin{array}{r}
\dot{r}=\frac{-1}{D} \sin (\phi-\theta)+\frac{1}{D}\left[\frac{\sin (\phi-\theta)}{1-\frac{2 r}{D} \cos (\phi-\theta)+\frac{r^{2}}{D^{2}}}\right], \\
\dot{\theta}=\frac{1}{r^{2}}+\frac{1}{r D} \cos (\phi-\theta)+\frac{1}{r D}\left[\frac{\frac{r}{D}-\cos (\phi-\theta)}{1-\frac{2 r}{D} \cos (\phi-\theta)+\frac{r^{2}}{D^{2}}}\right]
\end{array}
$$

where $\phi=\frac{2 t}{D^{2}}$ and the vortex period (defined as the time it takes for the two point vortices to complete one period), $\mathrm{T}$, is given by $T=D^{2} \pi$. As is evident from these formulas, as $D \rightarrow \infty$, the vortex period diverges like $O\left(D^{2}\right)$. The Hannay-Berry phase for this problem, denoted $\Delta \theta$, is defined to be the difference between the perturbed $(D<\infty)$ and the unperturbed $(D=\infty)$ angle variable at the end of one vortex period $\mathrm{T}$, hence:

$$
\Delta \theta=\theta_{D<\infty}(T)-\theta_{D=\infty}(T) .
$$

For this paper, we will refer to the Hannay-Berry phase (H-B phase) as the leading term in the asymptotic expansion for $\Delta \theta$, which we denote $\Delta \theta_{0}$ :

$$
\Delta \theta_{0} \equiv \lim _{D \rightarrow \infty}\left[\theta_{D<\infty}(T)-\theta_{D=\infty}(T)\right]
$$

By using a multi-scale perturbation approach for large $D$, it was shown in [15] that $\Delta \theta_{0}$ for the particle is given by $\pi$. In this paper we will describe in detail how this phase anholonomy arises, and what implications it has when estimating the long time growth rate of interfaces in two-dimensional incompressible turbulent flows. We first show how the $\mathrm{H}-\mathrm{B}$ phase arises as a non-uniform limit process in which an increasingly large number of vanishingly small terms accumulate to form a finite quantity.

In Fig. 2 we show the basic configuration, focusing on the difference between the perturbed and unperturbed particle orbits. We write $D=\frac{1}{\epsilon}$ and are interested in the limit $\epsilon \rightarrow 0$. As shown in Fig. 2, $\Delta \alpha(\epsilon)$ denotes the angle difference between the perturbed and unperturbed particle orbits on each particle cycle. The H-B phase is the total accumulated angle difference during one vortex period $T \sim \frac{1}{\epsilon^{2}}$ in the limit $\epsilon \rightarrow 0$. If $n(\epsilon)$ denotes the number of particle orbits during one vortex period, then clearly $n \rightarrow \infty$ as $\epsilon \rightarrow 0$, while $\Delta \alpha \rightarrow 0$. The leading term in the asymptotic expansion for the H-B phase is the product of the two quantities in the limit of vanishing $\epsilon$ :

$$
\Delta \theta_{0} \equiv \lim _{\epsilon \rightarrow 0}[(\Delta \alpha) n]=\pi
$$

Viewed in this way as a limit process, it is clear that to get a finite non-zero H-B phase requires a delicate balance between the strength of the force imposed on the particle by the distant vortex, and the rate of rotation of the slowly varying background field. An important point to draw from this result is that the unperturbed problem $(D=\infty)$ is fundamentally different from the limiting problem $D \rightarrow \infty$. If one is interested in predicting the particle position after long times $\left(t \sim D^{2}\right)$ for large $D$, the particle will end up approximately $180^{\circ}$ out of phase from where it would be in the 
case $D=\infty$. Furthermore, the larger $D$ becomes, the more exact this $180^{\circ}$ 'phase anholonomy' becomes.

It is interesting to point out that if the tracer particle in Fig. 1 is replaced by a point vortex of equal strength and circulation as the other two, the H-B phase is $\frac{2}{3} \pi$. Deriving this result is more involved than the corresponding result for the restricted three vortex problem since the vortex motion is no longer circular.

In preparation for the next section where we work out a detailed example, notice that for large $D$, keeping only the leading terms in (1), (2) gives the approximate system:

$$
\begin{array}{r}
\dot{r}=\frac{r}{D^{2}} \sin (2(\phi-\theta))+O\left(\frac{1}{D^{3}}\right), \\
\dot{\theta}=\frac{1}{r^{2}}-\frac{1}{D^{2}} \cos (2(\phi-\theta))+O\left(\frac{1}{D^{3}}\right) .
\end{array}
$$

\section{A modified problem with a Berry phase}

A simpler problem than the restricted three vortex problem, but one that contains many of the same features, including a non-trivial H-B phase is shown in Fig. 3. A particle with coordinates $(r, \theta)$ rotates around a point vortex of strength $\Gamma$ at the origin, with a uniform background flow of strength $V_{\infty}$ rotating slowly through one cycle. Since the uniform flow affects both the particle and the vortex in the same way, we impose the constraint that the vortex is fixed at the origin for a non-trivial effect of the background flow on the relative motion between particle and vortex. In the restricted three vortex problem such a constraint was unnecessary since the rotating background flow from the far away vortex is non-uniform. The equations for the particle in this flowfield are given by:

$$
\begin{aligned}
\dot{r} & =V_{\infty} \sin (\phi-\theta), \\
\dot{\theta} & =\frac{\Gamma}{2 \pi r^{2}}-\frac{V_{\infty}}{r} \cos (\phi-\theta) .
\end{aligned}
$$

To mimick the scaling for the restricted three vortex problem as shown in (6), (7), we take $V_{\infty}=\epsilon^{2}$, $\phi=2 \tau+\phi_{0}, \tau=\epsilon^{2} t, T=\frac{\pi}{\epsilon^{2}}, \Gamma=2 \pi$. Here, $\tau$ is the 'slow' time, $T$ is the period of the background flowfield and $\phi_{0}$ is the initial orientation of the background flow. If $\phi_{0}=0$, the uniform field initially points downward, similar to the farfield force due to the distant vortex in the restricted three vortex problem. It is worth pointing out that in the absence of the point vortex at the origin $(\Gamma=0)$, it is straightforward to show directly from the equations that :

$$
(\dot{r})^{2}+(\dot{\theta} r)^{2}=V_{\infty}^{2}
$$

This is a statement that the particle moves along a circular orbit, a result that is more familiar when written in cartesian coordinates.

Our goal in this section is to derive an expression for the leading term of the Hannay-Berry phase for this problem by using a multi-scale perturbation method, as was done in [15]. Our main result can be summarized in the following:

Theorem 1: For a particle with coordinates $(r, \theta)$ starting at $\left(R_{0}, 0\right)$, governed by the system (8), (9) with $V_{\infty}=\epsilon^{2}, \phi=2 \tau+\phi_{0}$, and $\Gamma=2 \pi$, the Hannay-Berry phase is given by:

$$
\Delta \theta_{0}=\left[\frac{2 \pi}{R_{0}}\right] \cos \left(\phi_{0}\right)
$$


Notice that the sign of the H-B phase depends on the initial orientation of the background field relative to the particle position. In particular, if the initial angle is $\phi_{0}=(2 n+1) \frac{\pi}{2}$, the leading term of the $\mathrm{H}-\mathrm{B}$ phase vanishes. To compute the H-B phase for this problem, we expand in powers of $\epsilon$, noting that $r$ and $\theta$ are functions of both the fast time ' $t$ ' and slow time ' $\tau$ ':

$$
\begin{aligned}
& r(t, \tau)=\sum_{j=0}^{\infty} \epsilon^{j} r_{j}(t, \tau), \\
& \theta(t, \tau)=\sum_{j=0}^{\infty} \epsilon^{j} \theta_{j}(t, \tau) .
\end{aligned}
$$

Accordingly, the ordinary time derivatives in (8), (9) are replaced with partial derivatives $\frac{d}{d t} \rightarrow$ $\frac{\partial}{\partial t}+\epsilon^{2} \frac{\partial}{\partial \tau}$. The initial conditions $r(0,0)=R_{0}$ and $\theta(0,0)=0$ (which are independent of $\epsilon$ ) imply that:

$$
\begin{array}{r}
r_{0}(0,0)=R_{0}, \\
\theta_{0}(0,0)=0, \\
r_{j>0}(0,0)=0, \\
\theta_{j>0}(0,0)=0 .
\end{array}
$$

Our notation is that all 'tilde' variables are purely functions of slow time $\tau$.

To leading order, this gives the equations:

$$
\begin{aligned}
O(1): \frac{\partial r_{0}}{\partial t} & =0 \\
\frac{\partial \theta_{0}}{\partial t} & =\frac{1}{r_{0}^{2}}
\end{aligned}
$$

Hence:

$$
\begin{aligned}
& r_{0}=\tilde{r}_{0}(\tau), \\
& \theta_{0}=\frac{t}{r_{0}^{2}}+\tilde{\theta}_{0}(\tau),
\end{aligned}
$$

with initial condition $\tilde{\theta}_{0}(0)=0$ and $\tilde{r}_{0}(0)=R_{0}$. The expression for the H-B phase comes from evaluating this term at the end of one period $\mathrm{T}$ :

$$
\Delta \theta_{0}=\tilde{\theta}_{0}(T) .
$$

To compute this we need to develop the expansion through $O\left(\epsilon^{4}\right)$. At next order we get:

$$
\begin{aligned}
O(\epsilon): \frac{\partial r_{1}}{\partial t} & =0 \\
\frac{\partial \theta_{1}}{\partial t} & =-\frac{2 r_{1}}{r_{0}^{3}} .
\end{aligned}
$$

In order to prevent linear growth in time for $\theta_{1}$, we must enforce the condition that $r_{1} \equiv 0$. This then allows us to conclude that $\theta_{1} \equiv \tilde{\theta}_{1}(\tau)$ with initial data $\tilde{\theta}_{1}(0)=0$.

At next order we get:

$$
\begin{aligned}
O\left(\epsilon^{2}\right): \frac{\partial r_{2}}{\partial t} & =-\frac{d \tilde{r}_{0}}{d \tau}+\sin \left(\left(\phi-\theta_{0}\right)\right) \\
\frac{\partial \theta_{2}}{\partial t} & =-\frac{\partial \theta_{0}}{\partial \tau}-\frac{2 r_{2}}{r_{0}^{3}}-\frac{1}{r_{0}} \cos \left(\left(\phi-\theta_{0}\right)\right)
\end{aligned}
$$


In order to prevent linear growth in time for $r_{2}$, we enforce the condition $\frac{d \tilde{r}_{0}}{d \tau}=0$. This gives $r_{0}=R_{0}$ from (14); hence $\theta_{0}=\frac{t}{R_{0}^{2}}+\tilde{\theta}_{0}(\tau)$ and $\frac{\partial \theta_{0}}{\partial \tau}=\frac{d \tilde{\theta}_{0}}{d \tau}$.

Solving (25) for $r_{2}$ gives $r_{2}=R_{0}^{2} \cos \left(\phi-\theta_{0}\right)+\tilde{r}_{2}(\tau)$, and therefore $\tilde{r}_{2}(0)=-R_{0}^{2} \cos \left(\phi_{0}\right)$. Proceeding to (26), it is clear after expanding all terms on the r.h.s. that to prevent linear growth in time for $\theta_{2}$, we must enforce the condition

$$
\frac{d \tilde{\theta}_{0}}{d \tau}=-2\left(\frac{\tilde{r}_{2}}{R_{0}^{3}}\right)
$$

This gives $\theta_{2}=3 R_{0} \sin \left(\phi-\theta_{0}\right)+\tilde{\theta}_{2}(\tau)$. The solution of $(27)$ for the initial condition $\tilde{\theta}_{0}(0)=0$ gives the H-B phase. To solve for $\tilde{r}_{2}$, we can proceed directly to the $O\left(\epsilon^{4}\right)$ equation in $r$ :

$$
\frac{\partial r_{4}}{\partial t}=-\frac{\partial r_{2}}{\partial \tau}-\frac{\tilde{\theta}_{1}^{2}}{2} \sin \left(\phi-\theta_{0}\right)-\theta_{2} \cos \left(\phi-\theta_{0}\right)
$$

Expanding the r.h.s., it is easy to see that the only term that can cause growth in time for $r_{4}$ is $\frac{d \tilde{r}_{2}}{d \tau}$. Hence, we set $\frac{d \tilde{r}_{2}}{d \tau}=0$ which gives the result $\tilde{r}_{2}=\tilde{r}_{2}(0)=-R_{0}^{2} \cos \left(\phi_{0}\right)$. Substituting this in $(27)$ and solving for $\tilde{\theta}_{0}$ with the initial condition $\tilde{\theta}_{0}(0)=0$, we get:

$$
\tilde{\theta}_{0}(\tau)=\left(\frac{2 \tau}{R_{0}}\right) \cos \left(\phi_{0}\right)=\left(\frac{2 \epsilon^{2} t}{R_{0}}\right) \cos \left(\phi_{0}\right) .
$$

Since $T=\frac{\pi}{\epsilon^{2}}$, the H-B phase, according to (22) is:

$$
\Delta \theta_{0}=\tilde{\theta}_{0}(T)=\frac{2 \pi}{R_{0}} \cos \left(\phi_{0}\right)
$$

\section{Slowly rotating spirals}

In this section we make contact with some work on spiral vortex models for 2D turbulence. Starting with the paper by Lundgren [11] and subsequently developed by Moffat [14] and Gilbert [9], the following point of view was advocated. $2 \mathrm{D}$ incompressible turbulence, in its simplest form, can be viewed as a collection of 'vortex spirals' which arise from the merging of a strong vortex core with a weak vortex patch. The weak vortex patch can be treated, to a first approximation, as a passively advected scalar field wrapping around the strong vortex core (see Fig. 1 from [9]). The result is a dynamic ensemble of interacting vortex spirals with a characteristic vorticity distribution, as discussed in Gilbert [9]. The presence of such structures in careful experiments has been commented on by Everson and Sreenivasan [7]. If one is interested in the 'diffusion' of vorticity in the spiral regions, or in the mixing of passive scalars in these regions as discussed, for example in $[1,13,16]$, the most relevant quantity to compute is the long time growth rate of the vorticity interface. We focus on that question in this section and show how this is intimately related to the H-B phase for the restricted three vortex problem. Hence, we analyse in this section the wrapping of a passive scalar interface around a point vortex in a slowly varying background field.

In Fig. 4 we show a sequence of snapshots of a scalar interface wrapping around a point vortex as time increases. The interface starts on a radial line segment along the $x$-axis, with initial length $\mathcal{L}_{u}(0)=|A-B|$. If $\xi$ is the interface parameter, then a particle at position $\xi$ on the interface at time $t=0$ has polar coordinates $(\rho(\xi, t), \phi(\xi, t))$ for $t>0$ given by $\rho(\xi, t)=\xi, \phi(\xi, t)=\frac{t}{\xi^{2}}$. We have 
normalized the vortex strength to unity. The interface length, as a function of time is given by:

$$
\begin{aligned}
\mathcal{L}_{u}(t) & =\int_{A}^{B} \sqrt{\left(\frac{d \rho}{d \xi}\right)^{2}+\left(\rho \frac{d \phi}{d \xi}\right)^{2}} d \xi \\
& =\int_{A}^{B} \sqrt{1+4 t^{2} \xi^{-4}} d \xi .
\end{aligned}
$$

From this, we can conclude that for short times, the interface grows like $\mathcal{L}_{u}(t) \sim \alpha t^{2}$, while for long times we get $\mathcal{L}_{u}(t) \sim \beta t$.

If a distant co-rotating vortex is placed a distance $D$ away, as in the restricted three vortex problem, the slowly varying background field will distort the interface as it wraps around the point vortex, hence affect the long time growth rate. In one vortex period $T \sim D^{2}$, the arclength will be corrected by a finite term which can be directly related to the H-B phase. In particular, we consider an asymptotic expansion for the arclength, $\mathcal{L}$, of the interface at the end of one vortex period T:

$$
\mathcal{L}(T) \sim \mathcal{L}_{0}(T)+O\left(\frac{1}{D}\right) .
$$

To compute $\mathcal{L}_{0}$, we identify the difference $\left(\mathcal{L}_{H B}\right)$ between the perturbed $(\mathcal{L})$ and unperturbed $\left(\mathcal{L}_{u}\right)$ arclengths from the following limit process:

$$
\mathcal{L}_{H B} \equiv \lim _{D \rightarrow \infty}\left[\mathcal{L}(T)-\mathcal{L}_{u}(T)\right]
$$

The main result, which we summarize here concerns a decomposition of the arclength into two distinct parts:

Theorem 2: During one vortex period $T \sim D^{2}$, the leading term in the asymptotic expansion for the arclength, $\mathcal{L}_{0}$, decomposes into two parts:

$$
\mathcal{L}_{0} \equiv \mathcal{L}_{u}+\mathcal{L}_{H B}
$$

The first term, $\mathcal{L}_{u}$, is given by the formula (32). This contribution is due to the wrapping of the interface around the vortex center. The second term is given by:

$$
\mathcal{L}_{H B}=-\pi|A-B|
$$

and is directly related to the H-B phase for the restricted three vortex problem.

While we don't prove the result here, several points should be emphasized:

1. Requiring that the background flow be slowly varying is natural if one is interested in the interfacial dynamics near a vortex core where the wrapping process is rapid compared to the dynamical time scale introduced by the other distant spirals.

2. The leading term giving the interfacial growth is due to two distinct contributions: one from the wrapping of the interface around the central vortex $\left(\mathcal{L}_{u}\right)$, the other from the slowly varying background field $\left(\mathcal{L}_{H B}\right)$. For long times $T \sim D^{2}$, the contribution due to the slowly rotating background field accumulates to a finite amount which can be directly related to the H-B phase $\Delta \theta_{0}$ :

$$
\mathcal{L}_{H B} \equiv-\Delta \theta_{0} * \mathcal{L}(0)
$$

3. Note that the limit process $D \rightarrow \infty$ is crucial to the result. The limiting case $D=\infty$ corresponds to an interface wrapping around an isolated point vortex whose interface grows according to $(32)$, with no correction term $\mathcal{L}_{H B}$. 


\section{Conclusions}

Since the fundamental paper by Berry in 1984, 'phase anholonomies' have been found to play an important role in a wide variety of physical phenomena. The 'Berry phase' (or 'Hannay angle') is a special kind of anholonomy and as such has a purely geometric interpretation. As emphasized in this paper, however, the Berry phase also has a nice analytical interpretation as the leading term in a non-uniform asymptotic expansion. It plays an important role when analysing the long time growth of passive scalar interfaces, of the type considered in spiral vortex models of $2 \mathrm{D}$ turbulence [9]. We are currently applying these ideas to study the growth of interfaces in two dimensional shear layers during the roll up process to gain a quantitative understanding of how the 'vortex pairing' stage of the shear layer evolution affects interfacial growth rates and hence the mixing process [17].

\section{Acknowledgments}

This work was supported in part by grant NSF-DMS-94-00032 from the National Science Foundation.

\section{References}

[1] Aref, H., "Integrable, Chaotic, and Turbulent Vortex Motion in Two-Dimensional Flows," Ann. Rev. Fluid Mech., 15, pp. 345, 1983.

[2] Berry, M.V., "Quantal Phase Factors accompanying Adiabatic Changes," Proc. Roy. Soc. Lond., A392, pp. 45, 1984.

[3] Berry, M.V., "Classical Adiabatic Angles and Quantal Adiabatic Phase," J. Phys. A.: Math. Gen., 18, pp. 15-27, 1985.

[4] Berry, M.V., "Quantum Phase Corrections from Adiabatic Iteration," Proc. Roy. Soc. Lond., A414, pp. 31-46, 1987.

[5] Berry, M.V., "The Geometric Phase," Scientific American, December, 1988.

[6] Berry, M.V., "Anticipations of the Geometric Phase," Physics Today, December, 1990.

[7] Everson, R.M. and Sreenivasan, K.R., "Accumulation Rates of Spiral-Like Structures in Fluid Flows," Proc. Roy. Soc. Lond., A437, 1992.

[8] Geometric Phases in Physics, Advanced Series in Mathematical Physics, Vol. 5, edited by A. Shapere and F. Wilczek, World Scientific, 1989.

[9] Gilbert, A., "Spiral Structures and Spectra in Two-Dimensional Turbulence," J. Fluid Mech., 193, pp. 475, 1988.

[10] Hannay, J.H., "Angle Variable Holonomy in Adiabatic Excursion of an Integrable Hamiltonian," J. Phys. A: Math. Gen., 18, pp. 221-230, 1985.

[11] Lundgren, T., "Strained Spiral Vortex Model for Turbulent Fine Structure," Phys. Fluids, 25 (12), pp. 2193, 1982.

[12] Marsden, J., O’Reilly, O.M., Wicklin, F.J. and Zombro, B.W., "Symmetry, Stability, Geometric Phases, and Mechanical Integrators, Parts I, II," Nonlinear Science Today, 1 (1,2), 1991. 
[13] Meiburg, E., Newton, P.K., Raju, N. and Ruetsch, G., "Unsteady Models for the Nonlinear Evolution of the Mixing Layer," Phys. Rev. E, August, 1995.

[14] Moffat, H.K., "Simple Topological Aspects of Vorticity Dynamics," Proc. IUTAM Symp. on Turbulence and Chaotic Phenomena in Fluids, edited by T. Tatsumi, Elsevier, p. 223, 1984.

[15] Newton, P.K., "Hannay-Berry Phase and the Restricted Three Vortex Problem," Physica D79, p. 416, 1994.

[16] Rom-Kedar, V., Leonard, A. and Wiggins, S., "An Analytical Study of Transport, Mixing, and Chaos in an Unsteady Vortical Flow," J. Fluid Mech., 214, pp. 347, 1990.

[17] Winant, C.D. and Browand, F.K., "Vortex Pairing: The Mechanism of Turbulent Mixing Layer Growth at Moderate Reynolds Numbers," J. Fluid Mech., 63, pp. 237, 1974.

[18] Zwanziger, J.W., Koenig, M. and Pines, A., "Berry's Phase," Ann. Rev. Phys. Chem., 41, pp. $601,1990$. 


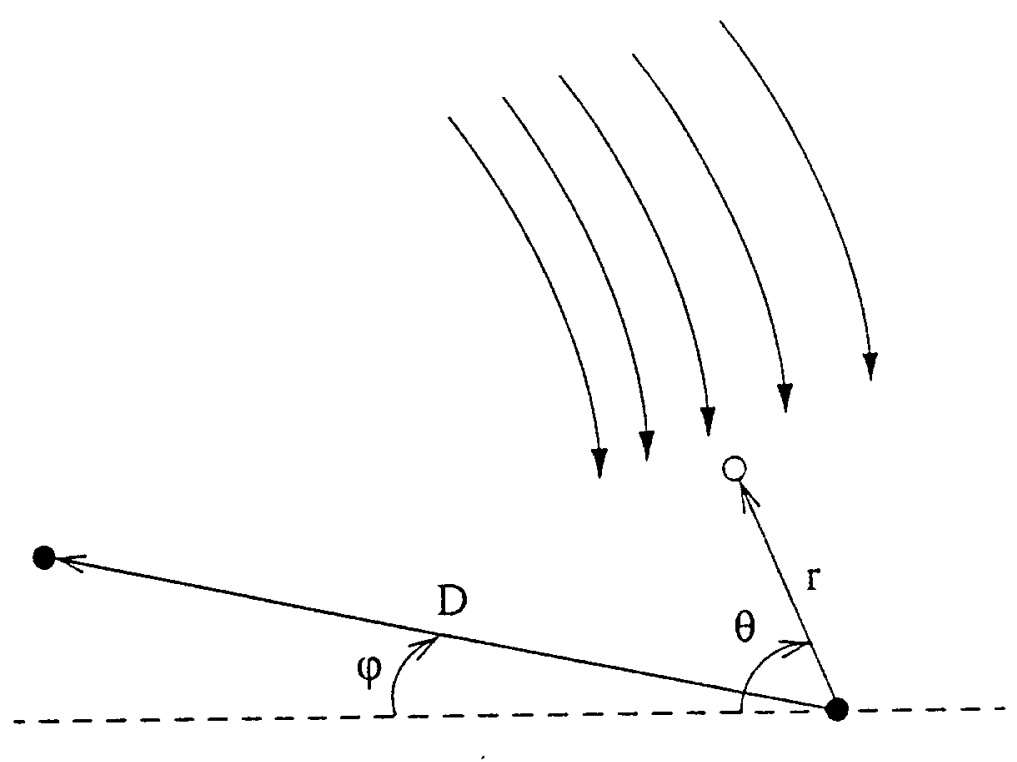

Figure 1: Configuration for restricted three vortex problem. Dark circles are equal strength vortices with clockwise circulation, light circle is a passive tracer particle. Forcefield on particle due to distant vortex is shown.

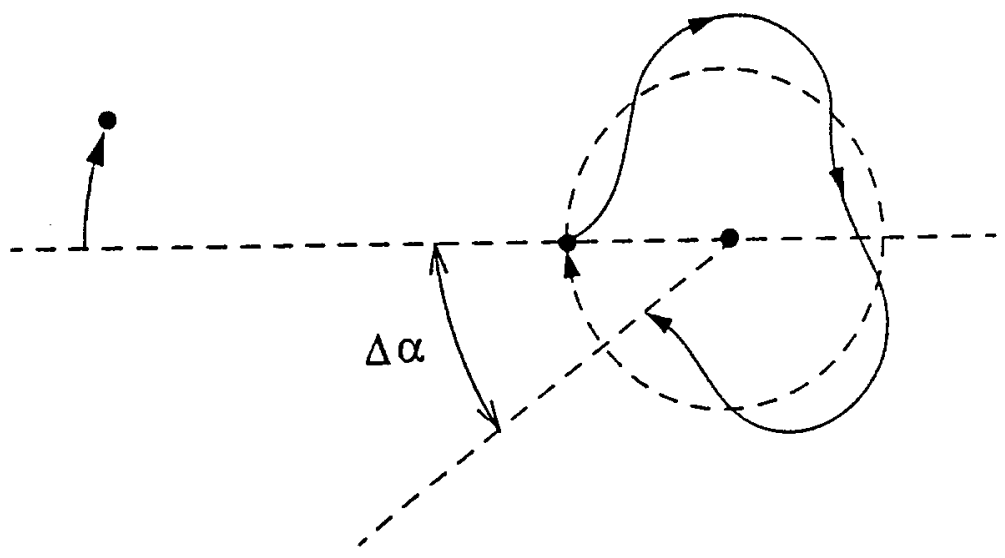

Figure 2: Comparison of angle variable for restricted three vortex problem. Dashed circle is the unperturbed $(D=\infty)$ particle trajectory. Solid curve is the perturbed $(D<\infty)$ particle trajectory. $\Delta \alpha$ marks the angle difference between the perturbed and unperturbed particle trajectories during one particle cycle. 


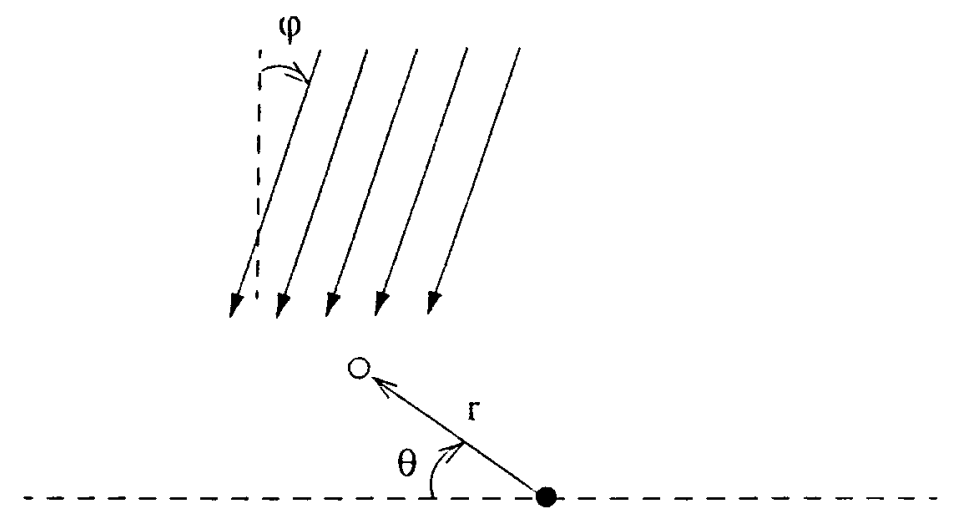

Figure 3: Configuration for modified problem in which a particle (light circle) rotates around a point vortex (dark circle) with a slowly rotating uniform background flowfield superimposed. When $\phi=0$, the background flow points downward.

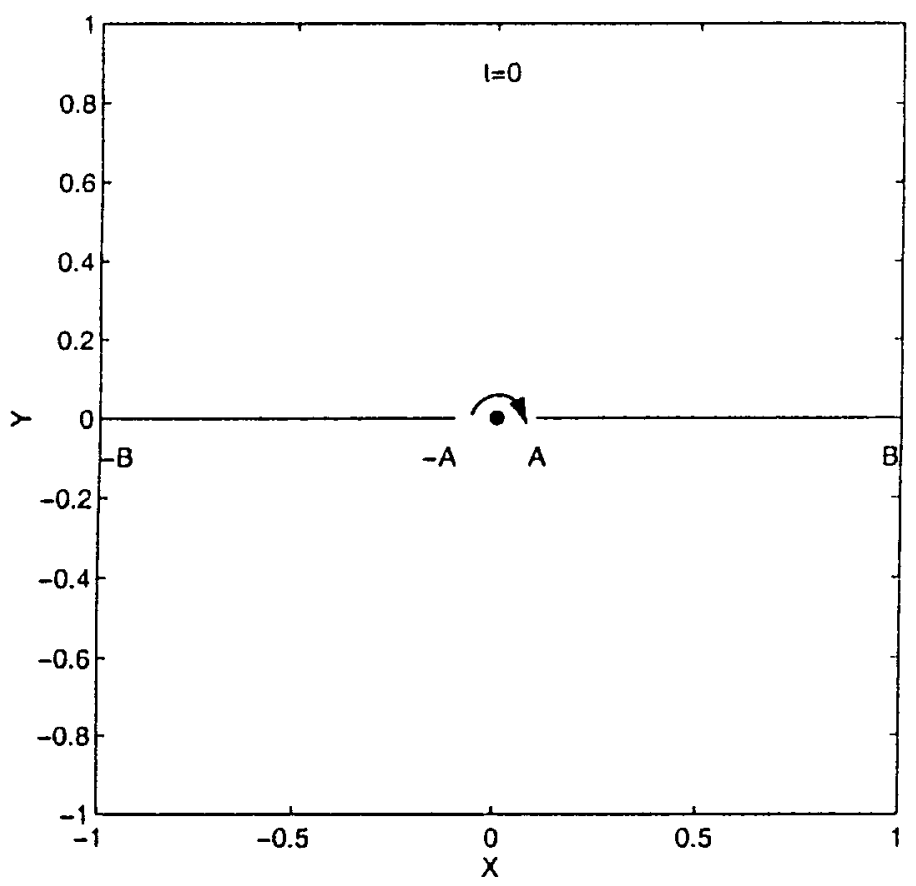

Figure 4: (a) Initial interface along horizontal axis from $[-B,-A],[A, B]$, with point vortex at center. 


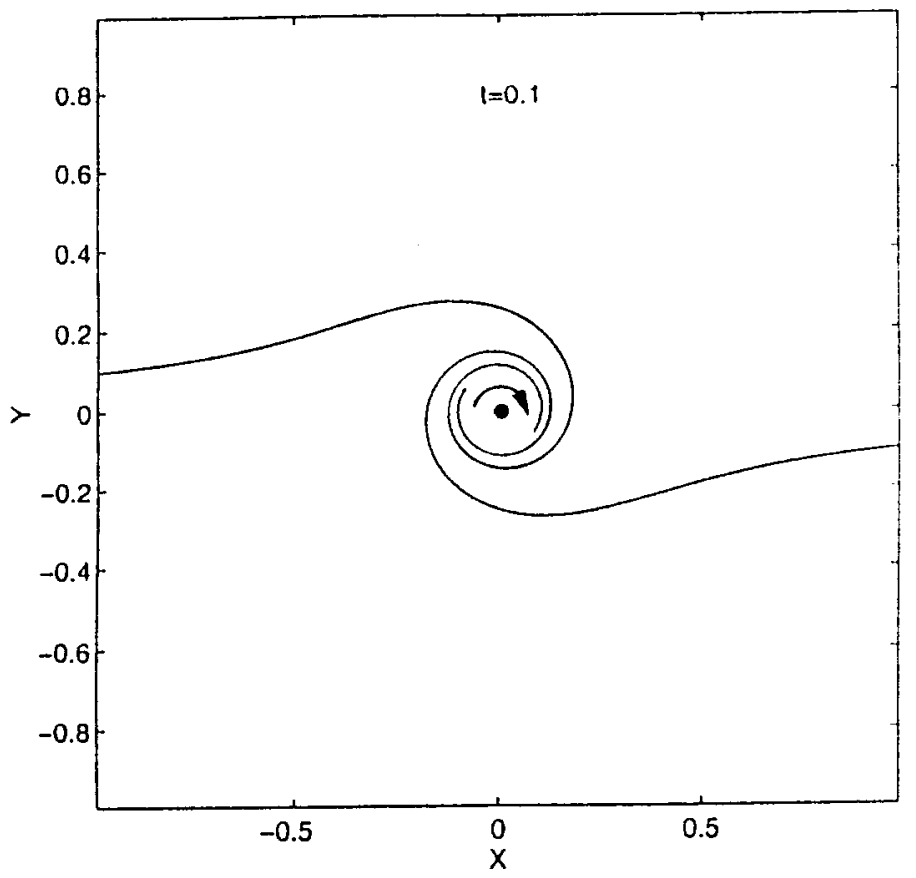

Figure 4: (b) Wrapping of interface around point vortex, $t=0.1$.

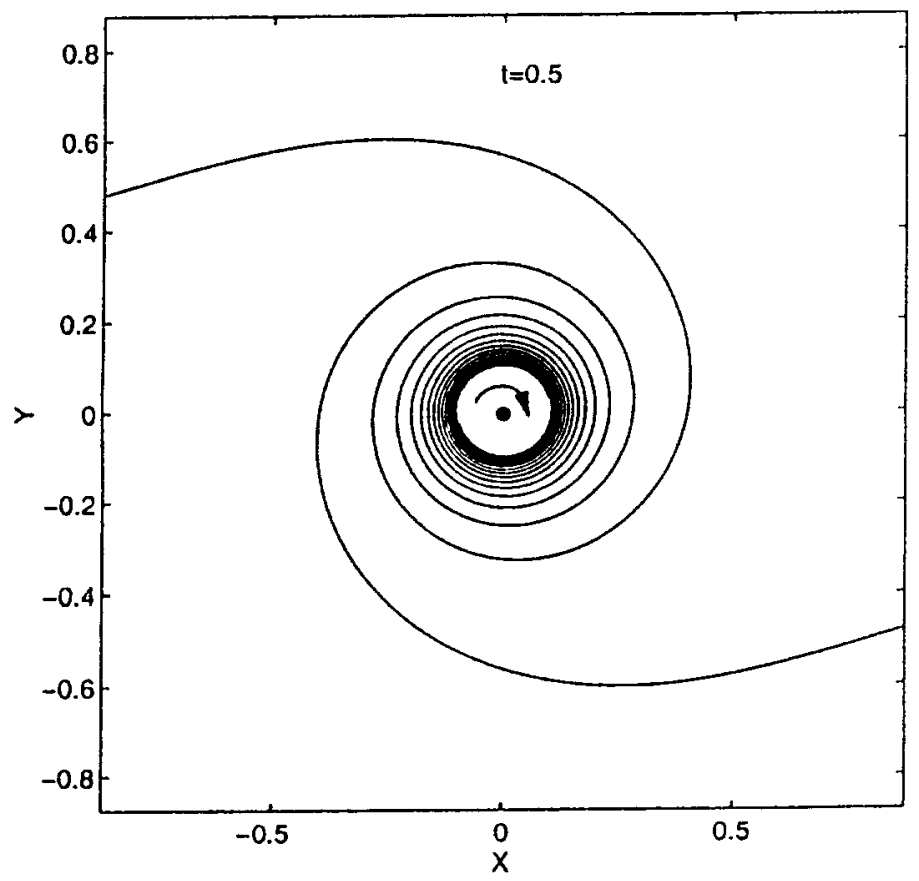

Figure 4: (c) Wrapping of interface around point vortex, $t=0.5$. 